\title{
Photonic crystal thin films of GaAs prepared by atomic layer deposition
}

\author{
I. M. Povey, D. Whitehead, K. Thomas, M. E. Pemble, ${ }^{\text {a) }}$ and M. Bardosova \\ Tyndall National Institute, University College Cork, Lee Maltings, Prospect Row, Cork, Republic of Ireland \\ J. Renard \\ Department of Materials Science and Technology, INSA, 20 Avenue des Buttes de Coësmes, \\ 35043 Rennes Cedex, France
}

(Received 19 December 2005; accepted 12 July 2006; published online 6 September 2006)

\begin{abstract}
Photonic crystal thin films were fabricated via the self-assembly of a lattice of silica spheres on silicon (100) substrates. Progressive infilling of the air spaces within the structure with GaAs was achieved using trimethylgallium and arsine under atomic-layer-deposition conditions. Samples with the highest levels of GaAs infill were subsequently inverted using selective etching. Reflectance spectra are interpreted via the Bragg expression and calculated photonic band structure diagrams. For GaAs infilled and inverted samples, the relative positions of the first and second order Bragg reflections are strongly influenced by the wavelength dependent refractive index. (C) 2006 American Institute of Physics. [DOI: 10.1063/1.2345359]
\end{abstract}

Since the pioneering work of Yablonovitch ${ }^{1}$ and John ${ }^{2}$ interest in photonic crystals has grown enormously. Photonic crystals can exhibit a photonic band gap where light possessing certain values of wave vector is not allowed to propagate in the material. Opals are natural examples of photonic band gap materials that may be readily synthesized in the laboratory. In such materials the nature of the band gap is not only influenced by the lattice parameters but also the contrast in the dielectric constant between the opal structure and its voids. This contrast can be enhanced by infilling the voids with a material of higher refractive index and still further by inversion, the selective removal of the host lattice. The effects of infiltrating semiconductor materials into opal have been demonstrated, for example, by the work of Astratov et al. with CdS nanoparticles, ${ }^{3}$ and Blanco et al. and Míguez et $a l$., who have shown that inverted opals made from $\mathrm{Si}$ or $\mathrm{Ge}$ possess a complete photonic gap, across the whole Brillouin zone. ${ }^{4,5}$ Materials that possess complete gaps may be particularly suited to the fabrication of novel filter or mirror-based devices, although the existence of well-defined "pseudogaps" in these materials, which effectively prevent light propagation in certain directions and not others, may also be of high technological significance, for example, in terms of waveguiding. ${ }^{6}$

Infilling the void spaces within host photonic crystal lattices has been demonstrated with a wide range of materials using methods such as wet chemical impregnation, ${ }^{7}$ chemical vapor deposition ${ }^{8-10}$ (CVD), and, recently, atomic layer deposition (ALD). The ALD technique was demonstrated for the $\mathrm{ZnO},{ }^{11} \mathrm{TiN}^{12}$ and $\mathrm{TiO}_{2}$ and $\mathrm{ZnS}: \mathrm{Mn}$ (Refs. 13 and 14) systems and shown to produce uniform and highly conformal infill from which inverted opals were fabricated with good photonic properties. In this present study we extend the ALD methodology to III/V materials and, in particular, GaAs.

GaAs-based photonic crystals are of particular interest in terms of both the nature of the photonic gap produced in a high refractive index contrast medium and the possibility of coupling these effects with both linear and nonlinear optical processes such as second harmonic generation, which would

${ }^{a)}$ Electronic mail: martyn.pemble@tyndall.ie be allowed in a material such as GaAs as a result of the direct band gap and the noncentrosymmetric nature of the bulk unit cell, respectively. ${ }^{15}$ To date while several two dimensional photonic band gap structures have been fabricated using lithographic methods, reports of fabrication of three dimensional GaAs photonic crystals are limited ${ }^{7}$ and there are no reports of growth by CVD or related methods.

In this present work we demonstrate that ALD methods based upon the use of a conventional metal organic vapor phase epitaxy (MOVPE) reactor may be used with a high degree of control to infill opal photonic crystals with GaAs. Furthermore the extent and uniformity of infill is such that subsequently, the opal host may be removed, creating a high quality GaAs inverse opal. We demonstrate that the optical properties of the resulting material may be understood via the Bragg equation which generally holds for such materials, provided that accurate refractive index and extinction coefficient values matched to the wavelength of features measured in the reflectance spectra are employed. In further support of the applicability of this simple Bragg model, we show that the observed behavior is consistent with that predicted via the modeling of the photonic band structure using the MIT photonic band (MPB) package. ${ }^{16}$

Artificial opal thin films were assembled on silicon (100) substrates by controlled evaporation, utilizing monodispersed silica spheres (260 $\mathrm{nm}$ in diameter after sintering) prepared by the Stöber synthesis ${ }^{17}$ Specular reflection measurements and electron microscopy confirm that the prepared opals exhibit high levels of uniformity. GaAs growth was performed in a horizontal reactor system (Thomas Swan Scientific Equipment single 2 in. wafer horizontal MOVPE reactor) maintained at $400{ }^{\circ} \mathrm{C}$ and 200 torr using trimethylgallium $(\mathrm{TMGa})$ and arsine $\left(\mathrm{AsH}_{3}\right)$ as precursors. Alternating gas pulses of TMGa $(3 \mathrm{~s})$ and $\mathrm{AsH}_{3}(8 \mathrm{~s})$ at flow rates of $5.3 \times 10^{-5}$ and $4.5 \times 10^{-3} \mathrm{~mol} \mathrm{~min}^{-1}$, respectively, were separated by a hydrogen purge $(2 \mathrm{~s})$ to prevent the reactant gases from mixing in the gas phase. Abrupt switching of precursors was achieved using a pressure-balanced run-and-vent gas manifold. The flow rate of hydrogen through the reactor was $16 \mathrm{dm}^{3} \mathrm{~min}^{-1}$ throughout precursor pulsing and purging. 

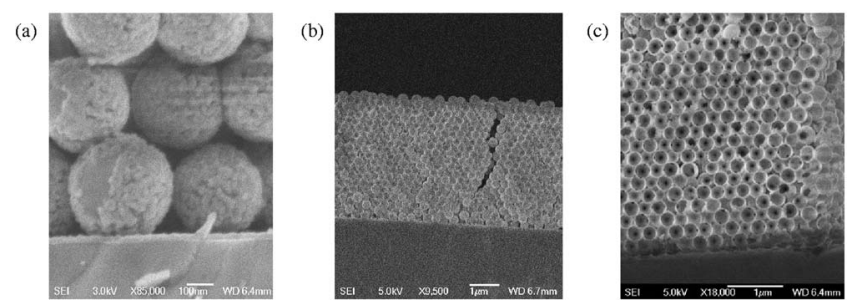

FIG. 1. SEM images of cleaved synthetic opals after (a) partial infill (200 cycles), (b) near total infill (800 cycles), and (c) inversion.

All samples were sintered under growth conditions prior to infiltration employing a $15 \mathrm{~min}$ temperature ramp designed to bring the substrate to growth temperature where it was held for a further 15 min prior to growth. The level of GaAs infill was tuned using an iterative process, varying the number of pulse sequences and calculating the infill using the Bragg expression. Inversion of infilled opal structures was achieved by immersion in 1\% HF solution for $20 \mathrm{~min}$. Optical characterization was performed by diffuse reflectance measurements, using a Brüker IFS66 spectrometer equipped with a variable angle reflectance stage, and Raman spectroscopy, using a Spectra Physics 2080/20s argon ion laser $(514.5 \mathrm{~nm})$ and a charged coupled device camera mounted on a Dilor XY 800 triple grating spectrometer.

Figure 1 shows SEM images recorded after 200 and 800 ALD cycles, showing that the infilling of the voids between the silica spheres in the opal structure is initially rough but conformal and virtually complete after 800 cycles. The crystalline nature of the infilled material is demonstrated by the measured Raman spectra in Fig. 2 via the presence of the TO $\left(263 \mathrm{~cm}^{-1}\right)$ and LO $\left(286 \mathrm{~cm}^{-1}\right)$ phonons for GaAs. The assignment is supported by the presence of weak but characteristic crystalline GaAs features in the x-ray diffraction spectra. Figure 3 shows reflectance data for the bare opal sample and after varying numbers of ALD cycles. This figure reveals that for the bare opal the Bragg peak as measured at an angle of incidence of $12^{\circ}$ from normal occurs at ca. $592 \mathrm{~nm}$. As the number of ALD cycles is then increased, so the Bragg peaks shift progressively to higher wavelengths eventually reaching ca. $916 \mathrm{~nm}$ after some 800 cycles.

The position of the $n$th order Bragg peak $(\lambda)$ is related to the effective dielectric constant of the material $\left(\varepsilon_{\text {eff }}\right)$, the weighted sum of the dielectric constant components, the lattice parameter $\left(d_{111}\right)$, and the angle of incidence $\theta$ by the expression

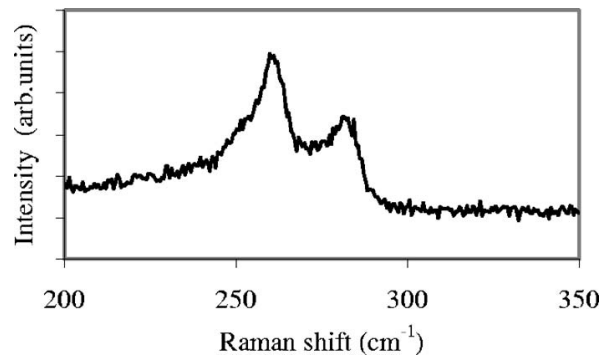

FIG. 2. Raman spectrum of an opal sample following 500 ALD cycles of GaAs growth, showing the TO and LO modes of bulk GaAs at 263 and

purely due to a second order process but the convolution of
Downloaded 08 Sep 2006 to 143.239.65.179. Redistribution subject to AlP license or copyright, see http://apl.aip.org/apl/copyright.jsp

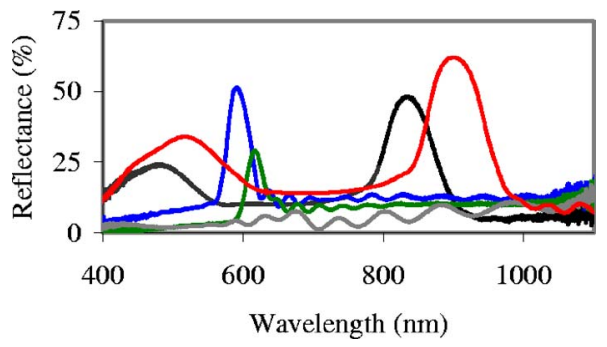

FIG. 3. (Color online) Diffuse reflectance spectra of opal thin films for (a) a bare opal (0\% infill) (blue line), (b) $15 \%$ infill (green line), (c) $26 \%$ infill (gray line), (d) $67 \%$ infill (black line), and (e) $91 \%$ infill of GaAs (red line). Note the progressive redshift of the first order Bragg peaks as the infill level increases. At higher levels of infill the partially unassigned secondary features shift into the accessible spectral window.

$$
\begin{aligned}
n \lambda= & 2 d_{111}\left\{\left(\varepsilon_{\mathrm{eff}}\right)-\sin ^{2} \theta\right\}^{1 / 2}, \\
\varepsilon_{\mathrm{eff}}= & f\left(n_{\mathrm{SiO}_{2}}+i k_{\mathrm{SiO}_{2}}\right)^{2}+f^{\prime}\left(n_{\mathrm{infill}}+i k_{\mathrm{infill}}\right)^{2}+f^{\prime \prime}\left(n_{\mathrm{air}}\right. \\
& \left.+i k_{\mathrm{air}}\right)^{2} \approx\left(n^{2}-k^{2}\right)_{\mathrm{SiO}_{2}}+\left(n^{2}-k^{2}\right)_{\mathrm{infill}}+\left(n^{2}-k^{2}\right)_{\mathrm{air}},
\end{aligned}
$$

where $n_{x}$ and $k_{x}$ are the refractive index and extinction coefficient of the component $x$, respectively.

Using Eqs. (1) and (2) and $n$ and $k$ values of 3.57 and 0.00 , respectively, it is possible to estimate that after 800 cycles, the contribution to the composite dielectric constant from air is approximately $9 \%$. Thus it may be concluded that the voids between the silica spheres are more than $90 \%$ infiltrated with GaAs. It should be noted that due to geometric considerations it is generally accepted that conformal growth within a face-centered-cubic opal structure will create air pockets within the voids that occupy $3.6 \%$ of the available space thus making the maximum theoretical infill 96.4\%. The spectrum obtained after some 200 cycles shows no Bragg peak. This result is a convenient way of demonstrating the photonic crystal nature of these samples, because the levels of GaAs infill for this sample are such that the weighted average dielectric constant of the material occupying the voids in the opal, i.e., $\left(n^{2}-k^{2}\right)_{\mathrm{GaAs}}+\left(n^{2}-k^{2}\right)_{\text {air }}$, is now equal to the dielectric constant of the silica. Thus this material possesses no refractive index contrast and as such the Bragg reflection is absent. From Eqs. (1) and (2) it may be estimated that the level of infill at this point is ca. $26 \%$. Peak positions, absolute reflectivity, and calculated levels of infill with their corresponding values of $n$ and $k$ are tabulated in Table I for a range of ALD cycle numbers.

Also of note is the appearance of lower wavelength features in the spectra for sample produced with higher levels of infill. These features could be assigned as second order peaks of the Bragg reflection, i.e., when $n=2$ in Eq. (1). However, the shift from the simple $2 \lambda$ relationship predicted by the Bragg equation can only be partially explained by the significant variation in refractive index and extinction coefficient of GaAs with wavelength, varying from $n=4.4$ to 3.5 and $k=2.0$ to 0.0 across the region studied $(400-950 \mathrm{~nm}){ }^{18,19}$ Furthermore, when infiltration calculations between the first and proposed second order features are compared (Table I) there is considerable disparity, with the second order features consistently overestimating the infill. This leads to the conclusion that the features are not purely due to a second order process but the convolution of AIP license or copyright, see http://apl.aip.org/apl/copyright.jsp 
TABLE I. Positions of the Bragg peaks measured by reflectance spectroscopy for samples after different numbers of ALD cycles. The corresponding values of $n$ and $k$ for GaAs and the percentage levels of infill estimated from these parameters are also given.

\begin{tabular}{rccccccccc}
\hline \hline $\begin{array}{c}\text { ALD } \\
\text { cycles }\end{array}$ & $\begin{array}{c}\text { First order } \\
\text { Bragg peak } \\
(\mathrm{nm})\end{array}$ & $n^{\mathrm{a}, \mathrm{b}}$ & $k^{\mathrm{a}, \mathrm{b}}$ & $\begin{array}{c}\text { Estimated } \\
\text { infill } \\
(\%)\end{array}$ & $\begin{array}{c}\text { Absolute } \\
\text { reflectivity } \\
(\%)\end{array}$ & $\begin{array}{c}\text { Secondary } \\
\text { peak } \\
(\mathrm{nm})\end{array}$ & $n^{\mathrm{a}, \mathrm{b}}$ & $k^{\mathrm{a}, \mathrm{b}}$ & $\begin{array}{c}\text { Estimated } \\
\text { infill } \\
(\%)\end{array}$ \\
\hline 0 & 592 & & & 0 & 51 & & & & \\
100 & 616 & 3.86 & 0.211 & 15 & 29 & & & & \\
200 & & 3.77 & 0.165 & 26 & & & & & \\
500 & 840 & 3.62 & 0.08 & 67 & 48 & 488 & 4.396 & 0.434 & 70 \\
800 & 916 & 3.57 & 0.00 & 91 & 62 & 540 & 4.087 & 0.286 & 106 \\
\hline \hline
\end{tabular}

${ }^{a}$ Reference 18 .

${ }^{\mathrm{b}}$ Reference 19.

more than one band. This argument is supported by the broadness and hints of structure exhibited by these spectral features.

Turning now to a comparison between experimental result and theory, Fig. 4 shows the photonic Brillouin zone predicted for a GaAs inverted structure calculated using the MIT photonic band package ${ }^{16}$ using a face-centered-cubic lattice with $260 \mathrm{~nm}$ spheres, $n=3.62$, and $k=0.0$. The singular refractive index value used in the calculations was chosen to match the Bragg reflection peak observed for the inverted structure. The calculated Brillouin zone can be directly compared to reflection data displayed in Fig. 4 from which it is evident that there is good agreement with the position of the first order Bragg reflection and the pseudogap between the second and third bands (between the $U$ and $L$ points in the Brillouin zone). The broad spectral feature at ca. 6 $\times 10^{14} \mathrm{~Hz}$, which is unlikely to be purely a second order Bragg peak, could have a contribution from a full gap between the eighth and ninth bands. This argument is supported by the presence of a shoulder on the broad secondary feature at ca. $4.5 \times 10^{14} \mathrm{~Hz}$ that can be attributed to a pseudogap between the fourth and fifth bands (between the $U-L$ and $\Gamma-L$ points in the Brillouin zone). However, assignments of these higher order bands should be treated with caution as the dispersions in $n$ and $k$ are not considered at these wavelengths

In this letter we have described the production and characterization of the first CVD grown GaAs silica opal structure by employing an ALD protocol. It has been demonstrated that ALD is an excellent method for the tunable
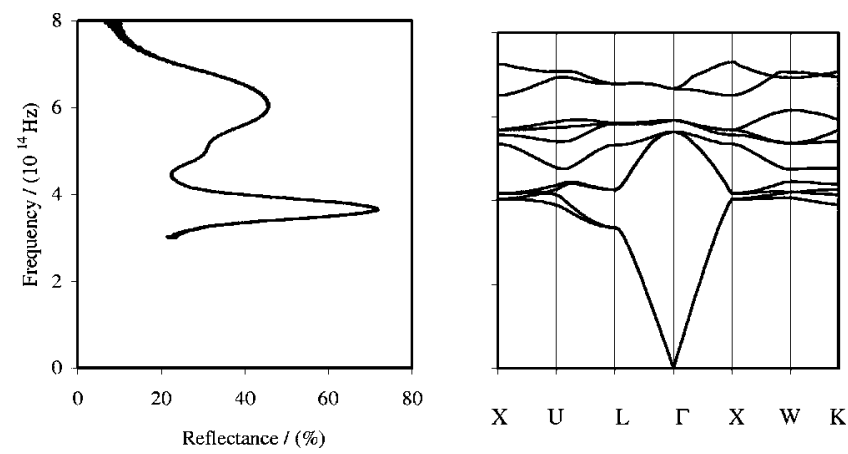

FIG. 4. Comparison of the measured reflectance spectrum of an inverted GaAs opal and the corresponding calculated Brillouin zone. infiltration of GaAs into silica opal thin films, uniformly penetrating throughout the structure to give fractional infill of up to ca. $91 \%$ of the available space. Optical measurements confirm the level of infill and nature of the material. In addition, for an inverted GaAs opal, comparison of the optical measurements with theoretical calculations demonstrates reasonable agreement with the predicted band structure, although unambiguous identification of higher energy features has not yet been achieved.

The authors would like to thank M. Schmidt for acquiring the Raman spectra and R. H. Tredgold for helpful discussions. This work was supported by Science Foundation Ireland.

${ }^{1}$ E. Yablonovitch, Phys. Rev. Lett. 58, 2059 (1987).

${ }^{2}$ S. John, Phys. Rev. Lett. 58, 2486 (1987).

${ }^{3}$ V. N. Astratov, V. N. Bogomolov, A. A. Kaplyanskii, A. V. Prokofiev, L. A. Samoilovich, S. M. Samoilovich, and Y. A. Vlasov, Nuovo Cimento D 17, 1349 (1995)

${ }^{4}$ A. Blanco, E. Chomski, S. Grabtchak, M. Ibisate, S. John, S. W. Leonard, C. Lopez, F. Meseguer, H. Miguez, J. P. Mondia, G. A. Ozin, O. Toader, and H. M. van Driel, Nature (London) 405, 437 (2000).

${ }^{5}$ H. Míguez, E. Chomski, F. Garcia-Santamaria, M. Ibisate, S. John, C. Lopez, F. Meseguer, J. P. Mondia, G. A. Ozin, O. Toader, and H. M. van Driel, Adv. Mater. (Weinheim, Ger.) 13, 1634 (2001).

${ }^{6}$ M. T. Todaro, T. Stomeo, V. Vitale, M. DeVittorio, A. Passaseo, R. Cingolani, F. Romanato, L. Businaro, and E. Di Fabrizio, Microelectron. Eng. 67-68, 670 (2003).

${ }^{7}$ Y. C. Lee, T. J. Kuo, C. J. Hsu, Y. W. Su, and C. C. Chen, Langmuir 18, 9942 (2002).

${ }^{8}$ H. M. Yates, M. E. Pemble, A. Blanco, H. Miguez, C. Lopez, and F. Meseguer, Chem. Vap. Deposition 6, 283 (2000).

${ }^{9}$ H. M. Yates, M. E. Pemble, H. Miguez, A. Blanco, C. Lopez, F. Meseguer, and L. Vazquez, J. Cryst. Growth 193, 9 (1998).

${ }^{10}$ T. Chunhua, F. Guanghan, Z. Tianming, L. Shuti, and S. Huiqing, Physica B 363, 1 (2005).

${ }^{11}$ M. Scharrer, X. Wu, A. Yamilov, H. Cao, and R. P. H. Chang, Appl. Phys. Lett. 86, 151113 (2005).

${ }^{12}$ A. Rugge, J. S. Becker, R. G. Gordon, and S. H. Tolbert, Nano Lett. 3, 1293 (2003).

${ }^{13}$ J. S. King, C. W. Neff, C. J. Summers, W. Park, S. Blomquist, E. Forsythe, and D. Morton, Appl. Phys. Lett. 83, 2566 (2003).

${ }^{14}$ J. S. King, D. Heineman, E. Graugnard, and C. J. Summers, Appl. Surf. Sci. 244, 511 (2005).

${ }^{15}$ A. Yariv, Quantum Electronics, 3rd ed. (Wiley, New York, 1989), p. 305.

${ }^{16}$ S. G. Johnson and J. D. Joannopoulos, Opt. Express 8, 173 (2001).

${ }^{17}$ W. Stöber, A. Fink, and E. Bohn, J. Colloid Interface Sci. 26, 62 (1968).

${ }^{18}$ H. Burkhard, H. W. Dinges, and E. Kuphal, J. Appl. Phys. 53, 655 (1982).

${ }^{19}$ S. Zollner, J. Appl. Phys. 90, 515 (2001). 